\title{
Acceptance of Illness, Satisfaction with Life, Sense of Stigmatization, and Quality of Life among People with Psoriasis: A Cross-Sectional Study
}

\author{
Beata Kowalewska (D) - Mateusz Cybulski (D) - Barbara Jankowiak (D) · \\ Elżbieta Krajewska-Kułak (D)
}

Received: March 4, 2020 / Published online: March 19, 2020

(C) The Author(s) 2020

\begin{abstract}
Introduction: Psoriasis is a complex disease with a heterogeneous presentation, which may have a profound effect on the everyday functioning of patients. As a strongly stressogenic disease, psoriasis may cause lack of illness acceptance and contribute to negative changes in self-image. Usually, psoriasis also has a detrimental effect on quality of life. The aim of this study is to determine the levels of illness acceptance, satisfaction with life, stigmatization, and quality of life in people with psoriasis, to analyze relationships among these variables, and to verify whether they are modulated by selected sociodemographic factors.

Methods: A total of 366 people with psoriasis treated at the Dermatology and Medical Cosmetology Clinic in Białystok were included. The study was conducted using a short survey prepared by the authors and five validated psychometric scales: Acceptance of Illness Scale (AIS), Satisfaction with Life Scale (SWLS), 6-item
\end{abstract}

Enhanced Digital Features To view enhanced digital features for this article go to https://doi.org/10.6084/ m9.figshare.11932881.

B. Kowalewska · M. Cybulski ( $₫) \cdot$ B. Jankowiak . E. Krajewska-Kułak

Department of Integrated Medical Care, Faculty of Health Sciences, Medical University of Białystok, M. Skłodowskiej-Curie 7A str., 15-096 Białystok, Poland e-mail: mateusz.cybulski@umb.edu.pl
Stigmatization Scale, 33-item Feelings of Stigmatization Questionnaire, and Dermatology Life Quality Index (DLQI).

Results: Median AIS score was 24 pts, which corresponds to a moderate level of illness acceptance. The level of illness acceptance among men was significantly higher than among women. More than half of respondents assessed their satisfaction with life as low. Men showed low levels of satisfaction with life slightly more often than women. Patients living in the countryside showed high levels of satisfaction with life significantly more often than those living in cities/towns. Mean scores for the 6-item Stigmatization Scale and the 33-item Feelings of Stigmatization Questionnaire were 8.73 pts and $90.06 \mathrm{pts}$, respectively, with no significant differences between the results of male and female patients. The DLQI scores for the study group ranged between 0 and 30 pts, with the mean value of 17.81 pts corresponding to a severe deterioration of quality of life. Statistically significant differences between quality of life and all sociodemographic variables were demonstrated.

Conclusions: People with psoriasis participating in this study showed moderate levels of illness acceptance and stigmatization, low level of satisfaction with life, and moderately deteriorated quality of life. Acceptance of illness was significantly modulated by patient sex. The level of illness acceptance among men was significantly higher than among women. 
Furthermore, illness acceptance exerted a significant effect on satisfaction with life in psoriasis patients. Place of residence exerted significant effects on satisfaction with life (higher in countryside dwellers), sense of stigmatization (stronger in countryside dwellers), and quality of life (more severely deteriorated in city/town dwellers). Disease duration significantly affected the degree of life satisfaction, sense of stigmatization (measured using a 33-item Feelings of Stigmatization Questionnaire), and quality of life among patients with psoriasis.

Keywords: Acceptance of illness; Healthrelated quality of life; Psoriasis; Quality of life; Satisfaction with life; Skin diseases; Stigmatization

\section{Key Summary Points}

\section{Why carry out this study?}

Psoriasis is a complex disease with heterogeneous presentation, which may have a profound effect on the everyday functioning of patients. As a strongly stressogenic disease, psoriasis may cause lack of illness acceptance and contribute to negative changes in self-image. Usually, psoriasis has also a detrimental effect on quality of life.

The aim of this study is to determine the levels of illness acceptance, satisfaction with life, stigmatization, and quality of life of people with psoriasis, to analyze relationships among these variables, and to verify whether they are modulated by selected sociodemographic factors.

\section{What was learned from the study?}

People with psoriasis participating in this study showed moderate levels of illness acceptance and stigmatization, low level of satisfaction with life, and moderately deteriorated quality of life.
Acceptance of illness was significantly modulated by patient sex. The level of illness acceptance was significantly higher among men than women. Furthermore, illness acceptance exerted a significant effect on satisfaction with life in psoriasis patients. Place of residence exerted significant effects on satisfaction with life (higher in countryside dwellers), sense of stigmatization (stronger in countryside dwellers), and quality of life (more severely deteriorated in city/town dwellers).

The lowest levels of illness acceptance, satisfaction with life, and quality of life, as well as the strongest sense of stigmatization, were found in patients with psoriatic lesions spread across the whole body.

\section{INTRODUCTION}

Psoriasis is a chronic disease of the skin that affects approximately $2 \%$ of the global population [1]. Primary manifestations of psoriasis include itchiness, pain, and presence of thick red scaling skin lesions that may develop in each body area [2-4].

As a complex disease with heterogeneous presentation, psoriasis may have a profound effect on the everyday functioning of patients [5]. As a disease affecting the skin, psoriasis may predispose the patients to psychological disorders, such as depression, anxiety, hostility, and negative self-image, during its active phase [6]. Moreover, psoriasis is a strongly stressogenic disease, and enhanced stress may contribute to the exacerbation of psoriatic symptoms. Psoriasis has a huge impact on the psychosocial functioning of patients [7]. The disease may distort self-image of an individual, lower selfesteem, and evoke feelings of shame and embarrassment. Often, patients have the impression of being unattractive to others [7]. Due to their visibility, psoriatic lesions stimulate negative responses on other people, which eventually contributes to lack of illness 
acceptance and negative self-image of patients [8]. Problems with acceptance of the illness may trigger negative emotions. Lack of adjustment to the disease is associated with the feeling of harm and the disruption of biopsychosocial balance [9]. A higher level of illness acceptance is, in turn, reflected by lesser utilization of avoidance strategies in coping with stress. Dermatological conditions, especially psoriasis, may cause isolation in interpersonal relationships [9]. Considering all of the above, this disease exerts an unfavorable effect on quality of life in people with psoriasis [10-12].

The aim of this study is to determine the levels of illness acceptance, satisfaction with life, stigmatization, and quality of life in people with psoriasis, to analyze relationships among these variables, and to verify whether they are modulated by selected sociodemographic factors (age, sex, place of residence, and duration of the disease). We assumed that the levels of illness acceptance, quality of life, and satisfaction with life in people with psoriasis were lower than in patients with other chronic skin conditions (e.g., alopecia areata, rosacea). Moreover, we hypothesized that people with psoriasis show high levels of stigmatization and the latter parameter increases proportionally to a decrease in illness acceptance, quality of life, and satisfaction with life.

\section{METHODS}

\section{Participants}

The study included a group of people with psoriasis treated at the Dermatology and Medical Cosmetology clinic in Białystok, owned by Dr. Piotr Aleksiejczuk, MD, PhD.

Aside from diagnosis of psoriasis, another inclusion criterion was written informed consent to participate in the study. Each respondent could withdraw from the study at any time without giving any specific reason.

The study participants were chosen by means of purposive sampling. To make the sample representative, the authors planned to collect at least 200 completed questionnaires; therefore, the number of distributed questionnaires was much higher $(n=500)$. As expected, not all questionnaires were returned or complete. A considerable proportion of patients eligible for the study withdrew before completing the questionnaire. The response and refusal rates were $73.20 \%$ (366 questionnaires) and $26.80 \%$ (134 questionnaires), respectively.

The study was carried out between June 2018 and September 2019. The respondents received hard copies of the surveys, which they completed (after being provided with detailed instructions) by themselves or with the aid of a member of the research team, at the doctor's office or at home. The respondents who decided to complete the survey at home were provided with an addressed envelope with a postage stamp to send back the questionnaire.

\section{Compliance with Ethics Guidelines}

The study protocol was approved by the Bioethics Committee of the Medical University in Białystok in line with the relevant Polish law (statute no. R-I-002/285/2018 of 28 July 2018). The study procedures were carried out in agreement with the Declaration of Helsinki, as revised in 2018, and good clinical practice guidelines. Written informed consent was obtained from all patients before enrollment.

\section{Measures}

The study was conducted using a short survey prepared by the authors and five validated psychometric scales: Acceptance of Illness Scale (AIS), developed by Felton et al. and adapted to Polish conditions by Juczyński; Satisfaction with Life Scale (SWLS), authored by Diener et al., in Polish adaptation by Juczyński; 6-item Stigmatization Scale by $\mathrm{Lu}$ et al., adapted to Polish conditions by Hrehorów et al.; 33-item Feelings of Stigmatization Questionnaire by Ginsburg and Link, in Polish adaptation by Hrehorów et al.; and Dermatology Life Quality Index (DLQI) by Finlay and Khan, in Polish adaptation by Szepietowski et al. 


\section{Survey Created by the Authors}

The survey prepared by the authors consisted of eight closed-ended multiple-choice questions with one or more correct answers. The questions referred to sociodemographic characteristics (sex, age, marital status, education, and place of residence) of the respondents. Moreover, the study participants were asked about the time elapsed since diagnosis of psoriasis, family history of the disease, and location of psoriatic lesions.

\section{Acceptance of Illness Scale (AIS)}

The statements included in the AIS refer to some specific problems and limitations associated with the respondent's health condition. Acceptance of illness is defined as the lack of negative responses and emotions associated with the condition. AIS can be used to measure illness acceptance in any condition. The scale consists of eight statements describing negative consequences of poor health, limitations imposed by the disease, lack of independence, dependence on others, and lowered self-esteem. Each statement is scored on a 5-point Likerttype scale, from 1 point ("completely agree") to 5 points ("completely disagree"). Complete agreement with a statement (1 point) reflects a poor adjustment to the disease and a strong psychological discomfort, whereas complete disagreement (5 points) corresponds to full acceptance of the illness. The overall AIS score can range between 8 and 40 points (pts). The higher the score, the higher the acceptance of the condition and the lesser the negative emotions associated with the disease. AIS scores below 20 pts are considered low, whereas those above 30 pts correspond to a high level of illness acceptance; values between 20 and 30 pts are classified as moderate levels of illness acceptance. An alternative way of interpretation is a comparison between the empirical scores and the results obtained in other groups of patients, also with different conditions. Cronbach's alpha for the scale is $0.82[13,14]$.

\section{Satisfaction with Life Scale (SWLS)}

This scale consists of five statements. The respondent is asked to assess to which degree each statement refers to his/her life using a 7-point scale, where 1-completely disagree, 2-disagree, 3-somewhat disagree, 4-neither agree nor disagree, 5-somewhat agree, 6agree, and 7-completely agree [13, 15].

The scores are summed up to obtain the overall SWLS score, being a measure of one's satisfaction with life. Overall score can range from 5 to $35 \mathrm{pts,} \mathrm{with} \mathrm{higher} \mathrm{scores} \mathrm{corre-}$ sponding to higher levels of satisfaction with life. Results are interpreted as follows:

- 5-9 pts-respondent is absolutely dissatisfied with his/her life,

- 10-14 pts-respondent is highly dissatisfied with his/her life,

- 15-19 pts-respondent is somewhat dissatisfied with his/her life,

- 20 pts-respondent is neither satisfied nor dissatisfied with his/her life,

- 21-25 pts-respondent is somewhat satisfied with his/her life,

- 26-30 pts-respondent is highly satisfied with his/her life,

- 31-35 pts-respondent is absolutely satisfied with his/her life.

Cronbach's alpha for the scale is 0.81 $[13,15]$.

\section{6-Item Stigmatization Scale}

The 6-item Stigmatization Scale consists of six multiple-choice questions to which respondents answer choosing one out of four possible options: "no," "sometimes," "very often," or "always." Responses are scored from 0 to 3 pts. Overall score can range from 0 pts, corresponding to lack of stigmatization, to $18 \mathrm{pts,}$ representing the maximum stigmatization level. Hence, the higher the score, the higher the stigmatization level $[16,17]$.

\section{3-Item Feelings of Stigmatization Questionnaire}

This scale consists of 33 questions, each with five possible responses: "definitely yes", "yes", "rather yes", "rather no", "no", and "definitely no". The answers are scored from 0 to 5 pts. Overall score can range from 0 pts, interpreted as lack of stigmatization, to $165 \mathrm{pts,}$ 
Table 1 Sociodemographic characteristics of study cohort

\begin{tabular}{|c|c|c|c|c|c|c|}
\hline \multirow[t]{2}{*}{ Sociodemographic variable } & \multicolumn{2}{|c|}{ Total $(N=366)$} & \multicolumn{2}{|c|}{ Women $(N=189)$} & \multicolumn{2}{|c|}{$\operatorname{Men}(N=177)$} \\
\hline & $N$ & $\%$ & $N$ & $\%$ & $N$ & $\%$ \\
\hline \multicolumn{7}{|l|}{ Age (years) } \\
\hline $18-30$ & 63 & 17.21 & 51 & 26.98 & 12 & 6.78 \\
\hline $31-40$ & 96 & 26.23 & 63 & 33.33 & 33 & 18.64 \\
\hline $41-50$ & 66 & 18.03 & 27 & 14.29 & 39 & 22.03 \\
\hline $51-60$ & 87 & 23.77 & 24 & 12.70 & 63 & 35.59 \\
\hline$>60$ & 54 & 14.74 & 24 & 12.70 & 30 & 16.95 \\
\hline \multicolumn{7}{|l|}{ Marital status } \\
\hline Married & 207 & 56.56 & 87 & 46.03 & 120 & 67.80 \\
\hline Single & 78 & 21.31 & 57 & 30.16 & 21 & 11.86 \\
\hline Divorced & 57 & 15.57 & 33 & 17.46 & 24 & 13.56 \\
\hline Widowed & 24 & 6.56 & 12 & 6.35 & 12 & 6.78 \\
\hline \multicolumn{7}{|l|}{ Education } \\
\hline Primary & 48 & 13.11 & 9 & 4.76 & 39 & 22.03 \\
\hline Vocational & 36 & 9.84 & 9 & 4.76 & 27 & 15.25 \\
\hline Secondary & 129 & 35.25 & 66 & 34.92 & 63 & 35.59 \\
\hline Higher & 153 & 41.80 & 105 & 55.56 & 48 & 27.13 \\
\hline \multicolumn{7}{|l|}{ Place of residence } \\
\hline Village & 93 & 25.41 & 48 & 25.40 & 45 & 25.42 \\
\hline Town/city & 273 & 74.59 & 141 & 74.60 & 132 & 74.58 \\
\hline \multicolumn{7}{|l|}{ Duration of disease (years) } \\
\hline Up to 5 & 114 & 31.15 & 84 & 44.44 & 30 & 16.95 \\
\hline $6-19$ & 144 & 39.34 & 69 & 36.51 & 75 & 42.37 \\
\hline 20 and more & 108 & 29.51 & 36 & 19.05 & 72 & 40.68 \\
\hline \multicolumn{7}{|l|}{ Family history of psoriasis } \\
\hline None & 123 & 33.61 & 60 & 31.75 & 63 & 35.59 \\
\hline Mother & 57 & 15.57 & 45 & 23.81 & 12 & 6.78 \\
\hline Father & 60 & 16.39 & 30 & 15.87 & 30 & 16.95 \\
\hline Siblings & 15 & 4.10 & 3 & 1.58 & 12 & 6.78 \\
\hline Other relatives (grandmother, grandfather, aunt, uncle) & 66 & 18.03 & 27 & 14.29 & 39 & 22.03 \\
\hline Unknown & 45 & 12.30 & 24 & 12.70 & 21 & 11.87 \\
\hline
\end{tabular}


Table 2 Distribution of duration of disease variable (in years)

\begin{tabular}{llllllll}
\hline Group & $\boldsymbol{N}$ & $\bar{x}( \pm$ SD) & Me & Min. (months) & Max. (months) & $Q_{1}$ & $Q_{3}$ \\
\hline Total & 366 & $14.18( \pm 11.79)$ & 11 & 1 & 51 & 5 & 20 \\
Women & 189 & $12.03( \pm 11.43)$ & 9 & 1 & 51 & 3.5 & 17 \\
Men & 177 & $16.48( \pm 11.75)$ & 12 & 5 & 47 & 6 & 25 \\
\hline
\end{tabular}

Max. maximum, $M e$ median, Min. minimum, $S D$ standard deviation, $Q_{1}$ lower quartile, $Q_{3}$ upper quartile, $\bar{x}$ mean

corresponding to the maximum stigmatization level. The higher the score for the 33-item scale, the higher the stigmatization level in the respondent. Additionally, this instrument measures the level of stigmatization in six domains: feeling of being rejected, feeling of being worse, vulnerability to others' opinions, guilt and shame, positive attitude, and secretiveness $[17,18]$.

\section{Dermatology Life Quality Index (DLQI)}

DLQI is one of the most commonly used instruments to examine the effect of skin conditions on quality of life. This scale is dedicated to adult patients, older than 16 years of age. It consists of 10 multiple-choice questions, each with one correct answer, referring to quality of life and emotions related to the presence of skin disease within a week preceding the survey. Each answer is scored on a 4-point Likert-type scale, from 0 to 3 . Overall score, being the sum of the scores for all individual questions, can range between 0 and 30 pts. The higher the score, the more deteriorated the quality of life of the respondent $[19,20]$. In 2005, a division into "point groups" was developed, prioritizing results obtained using this tool. The following five groups were distinguished: $0-1=$ no effect on patient's life, $2-5=$ small effect, $6-10=$ moderate effect, $11-20=$ very large effect, $21-30=$ extremely large effect [21].

\section{Statistical Analysis}

The results were subjected to statistical analysis with the Statistica 13.3 package (StatSoft, Poland). Statistical characteristics of discrete variables were presented as percentages, whereas characteristics of quantitative variables were shown as descriptive statistics (arithmetic means, standard deviations, medians, lower and upper quartiles, minimum and maximum values). The Shapiro-Wilk test was used to assess the normality of quantitative characteristics distribution. Since there were no normal distribution characteristics, the characteristics were analyzed using nonparametric methods. Two groups were compared using $U$ Mann-Whitney's test, and in case of more groups, ANOVA Kruskal-Wallis together test with post hoc tests. Pearson's chi-squared $\left(\chi^{2}\right)$ test was used to analyze the dependence of quality features. The strength and direction of the relationship among quantitative variables was determined using Spearman's correlation coefficient. The study results $p<0.05$ were regarded as statistically significant.

\section{RESULTS}

\section{Sociodemographic Characteristics of Respondents}

The study cohort consisted of 366 patients, including 189 women (51.64\%) and 177 men $(48.36 \%)$. The largest group of respondents (one-fourth of patients on average) was persons aged 31-40 years. Nearly one-fifth of participant belonged to the age group between 41 and 50 years. The majority of the respondents (more than 40\%) had higher education, approximately one-third of the patients had no more than secondary education, and $13 \%$ had vocational education. More than half of participants were married, one-fifth of respondents were single, and more than $15 \%$ were divorced. The distribution of places of residence was 


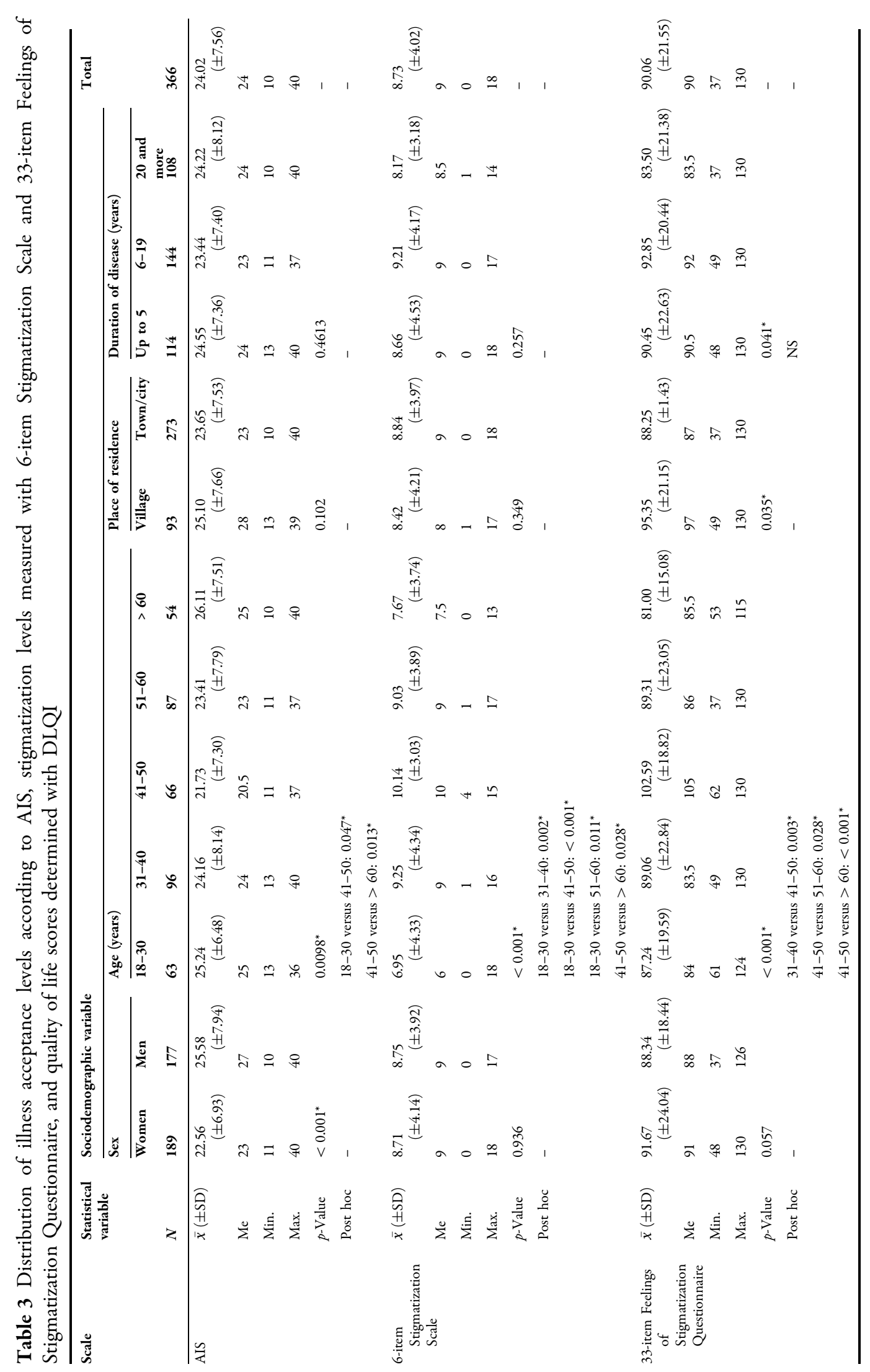




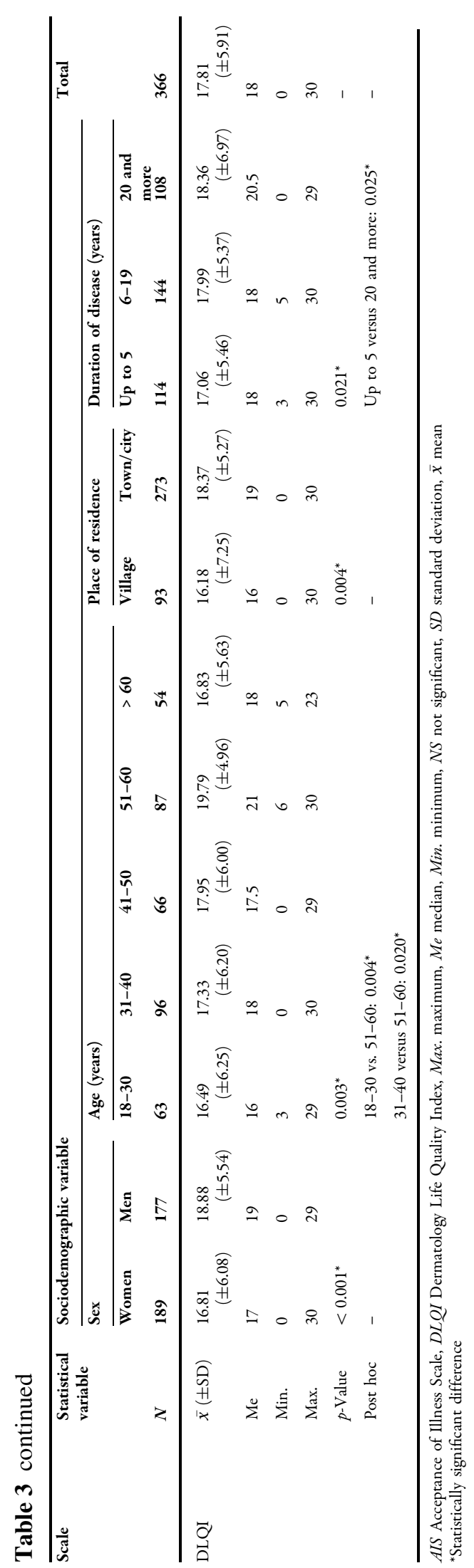

considerably skewed, as the vast majority of the study participants were city/town dwellers. The study subjects were distributed quite evenly in terms of psoriasis duration. In the largest proportion of female patients $(44.44 \%)$, the diagnosis was established within the last 5 years; in contrast, the percentage of male patients diagnosed with psoriasis within the last 5 years was no higher than $16.95 \%$. Up to $54.09 \%$ of respondents had a family history of psoriasis, most often in parents, followed by siblings and other relatives. Knowledge of family history of psoriasis among women and men was similar. The most often affected family members of female and male patients were their parents and other relatives, respectively. Psoriasis was diagnosed in both female $(23.77 \%)$ and male relatives $(29.51 \%)$ of the study participants. The occurrence of psoriasis among female relatives was more common among women, while men more often reported a history of psoriasis in their male relatives. Detailed sociodemographic characteristics of the respondents are presented in Table 1.

Mean duration of disease was 14.18 years $( \pm 11.82)$ and was shorter in female than in male patients. The study cohort included both patients diagnosed with psoriasis within the last 12 months and approximately 25\% of respondents with an at least 20-year history of disease (Table 2).

\section{Acceptance of Illness}

AIS scores ranged between 10 and 40 pts, with a mean value of 24.02 pts $( \pm 7.56)$, which corresponds to a moderate level of illness acceptance (Table 3). The level of illness acceptance among men was significantly higher than among women $(p<0.001)$. The lowest mean AIS scores were found in patients aged 41-50 years $(p=0.0098)$. The results for countryside dwellers were slightly higher than for city/town dwellers $(p=0.102)$. The level of illness acceptance did not differ considerably depending on time elapsed since diagnosis of psoriasis $(p=0.4613)$.

Most patients showed moderate levels of acceptance to psoriasis (Fig. 1). 


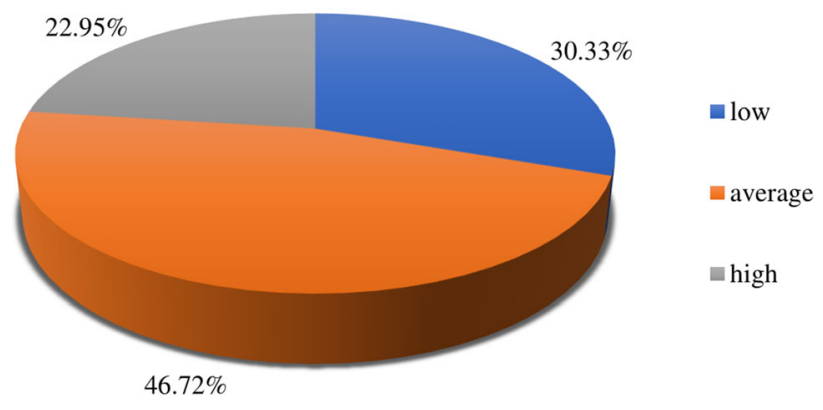

Fig. 1 Acceptance of psoriasis according to AIS. AIS Acceptance of Illness Scale

\section{Satisfaction with Life}

More than half of the respondents assessed their satisfaction with life as low (Table 4). Men showed low levels of satisfaction with life slightly more often than women $(p=0.282)$. Most $f$ requently, low levels of satisfaction with life were observed among 41-50-year-old patients $(p<0.001)$. Low and moderate SWLS scores were most common among patients in whom psoriasis had been diagnosed 6-19 years earlier $(p<0.001)$. Patients living in the countryside showed high levels of satisfaction with life significantly more often than those living in cities/towns $(p<0.001)$.

The vast majority of patients with low levels of illness acceptance also showed low levels of satisfaction with life; in contrast, low levels of satisfaction with life were found in only $28.6 \%$ of respondents with high levels of illness acceptance. Acceptance of the illness was shown to exert a significant effect on level of satisfaction with life with psoriasis $(r=0.535$, $p<0.001$ ) (Fig. 2).

\section{Sense of Stigmatization}

The mean score for the 6-item Stigmatization Scale was 8.73 pts $( \pm 4.02)$, with no significant differences in the results of male and female patients $(p=0.936) \quad$ (Table 3$)$. The lowest stigmatization levels were found in the youngest and oldest patients, 18-30-year-old and older than 60 years, respectively, and the highest mean scores were observed in the group of patients aged 41-50 years. Differences in stigmatization levels found in various age groups were statistically significant $(p<0.001)$. Patients living in cities/towns showed higher, albeit insignificantly, stigmatization levels than respondents living in the countryside $(p=0.349)$. Level of stigmatization in patients diagnosed with psoriasis 6-19 years earlier was higher than in other respondents, but this between-group difference also did not reach the threshold of statistical significance $(p=0.257)$.

The mean score for the 33-item Feelings of Stigmatization Questionnaire was 90.06 pts $( \pm 21.55)$, with no significant differences observed between men and women $(p=0.057)$ (Table 3). The lowest and highest stigmatization levels were observed in patients aged $>60$ years and 41-50 years. Age-related differences in this parameter were statistically significant $(p<0.001)$. The highest stigmatization levels were found in patients diagnosed with psoriasis 6-19 years earlier. Similar to age, duration of disease exerted a significant effect on this parameter $(p=0.041)$. According to the 33-item Feelings of Stigmatization Questionnaire scores, countryside dwellers showed significantly higher stigmatization levels than the city/town dwellers $(p=0.035)$.

\section{Quality of Life with Psoriasis}

DLQI scores for the present study cohort ranged between 0 and 30 pts, with a mean value of $17.81 \mathrm{pts}( \pm 5.91)$ corresponding to a severe deterioration of quality of life. However, the scores in nearly half of the respondents corresponded to a severe deterioration of quality of life (Fig. 3). 


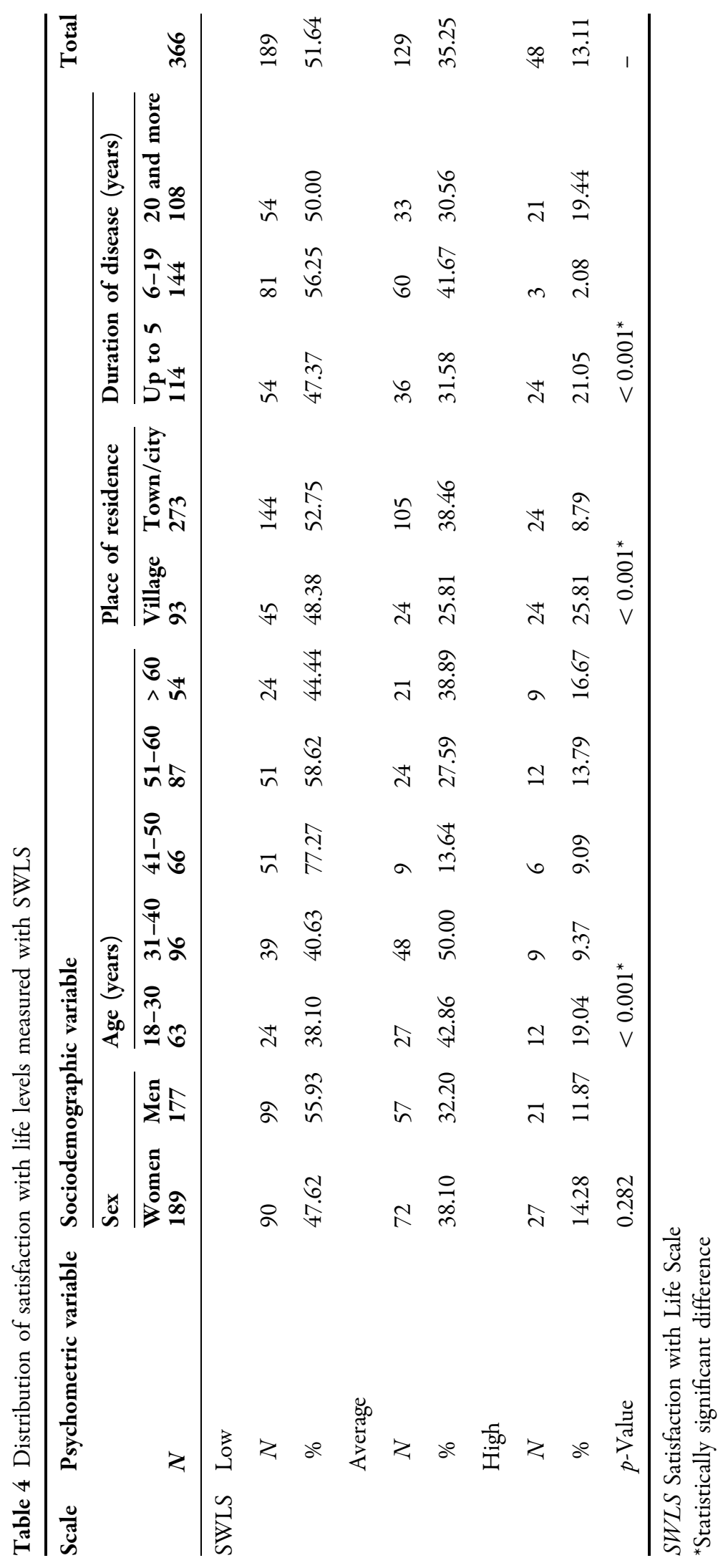




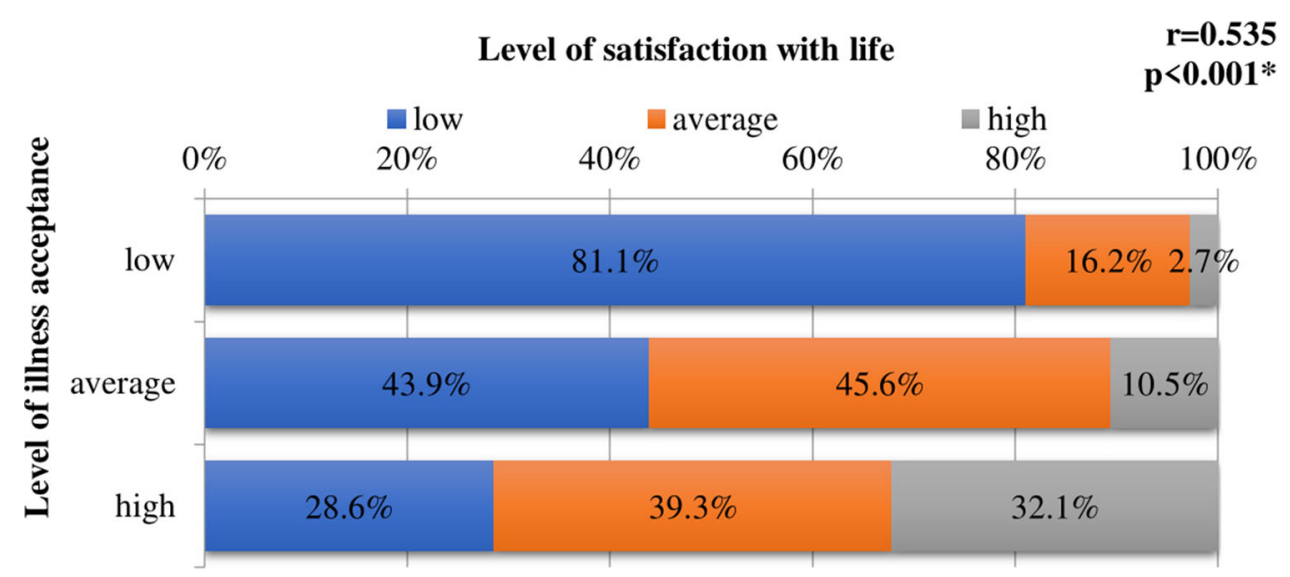

Fig. 2 Effect of illness acceptance on level of satisfaction with life. $r$ Spearman's correlation coefficient; *statistically significant correlation

Based on the distributions of the DLQI scores, men showed a moderate deterioration of quality of life twice as often as women. In turn, women more often than men had DLQI scores that corresponded to a normal quality of life or very severe deterioration of this parameter. Severe deterioration of quality of life was most often observed among patients aged $41-50$ years and $>60$ years. Regarding time elapsed since diagnosis of psoriasis, the most evident deterioration of quality of life was found in patients who were diagnosed with this disease $6-19$ years earlier. The sex- $(p<0.001)$, age- $(p=0.003)$, place of residence- $(p=0.004)$, and disease-duration-related $(p=0.025)$ differences in the distributions of DLQI scores were statistically significant (Table 3).

\section{Correlations between Scales}

As a result of the statistical analysis, no statistically significant correlations were found between DLQI values and the other examined scales. However, statistically significant positive high correlation coefficients between disease acceptance and life satisfaction as well as a sense of stigmatization measured using the 33-item Feelings of Stigmatization Questionnaire were found. The higher the level of acceptance of the disease, the higher the satisfaction with life and the lower the sense of stigmatization. A statistically significant negative correlation was also found between the sense of stigmatization measured using the 6-item Stigmatization Scale and the level of disease acceptance, life satisfaction and sense of stigmatization measured using the 33-item Feelings of Stigmatization Questionnaire, which means that the higher the sense of stigmatization, the lower the level of acceptance of disease and life satisfaction, and the higher the sense of stigmatization (measured with the 33-item Feelings of Stigmatization Questionnaire). Detailed values of correlation coefficients are presented in Table 5, and scatter plots in Fig. 4.

\section{DISCUSSION}

Psoriasis is a recurrent chronic disease having a devastating effect on quality of life and constituting a considerable burden for patients [22].

Similar to a previous study conducted by Jankowiak et al. [23], our female and male patients differed slightly in terms of their mean overall SWLS scores. Nevertheless, the largest proportion of respondents assessed their satisfaction with life as low. Satisfaction with life turned out to be the highest among the youngest patients (18-30 years). This observation is in opposition to the results published by Jankowiak et al. [23], as those authors found the highest levels of satisfaction with life in 50- and 60 -year-old patients. In our present study, the lowest SWLS scores were observed among 


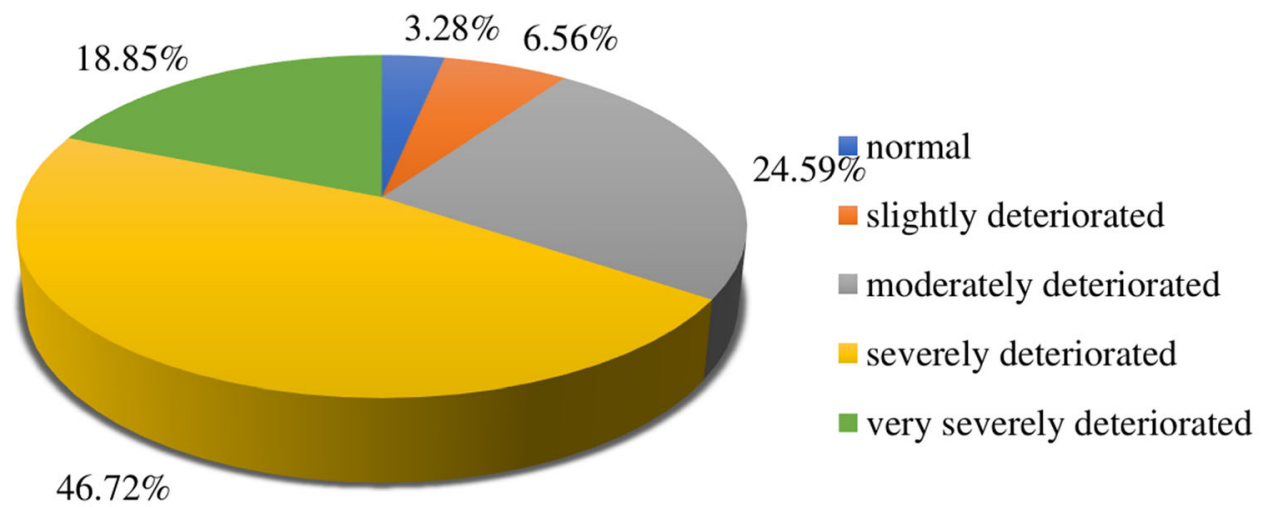

Fig. 3 Quality of life with psoriasis measured with DLQI. DLQI Dermatology Life Quality Index

Table 5 Correlation coefficients between standardized research instruments

\begin{tabular}{|c|c|c|c|c|}
\hline & DLQI & SWLS & 33-item Feelings of Stigmatization Questionnaire & 6-item Stigmatization Scale \\
\hline \multicolumn{5}{|l|}{ AIS } \\
\hline$r$ & -0.102 & 0.535 & 0.476 & -0.496 \\
\hline$p$-Value & 0.052 & $<0.001^{*}$ & $<0.001^{*}$ & $<0.001^{*}$ \\
\hline \multicolumn{5}{|c|}{ 6-item Stigmatization Scale } \\
\hline$r$ & -0.018 & -0.508 & -0.561 & - \\
\hline$p$-Value & 0.725 & $<0.001^{*}$ & $<0.001^{*}$ & - \\
\hline \multicolumn{5}{|c|}{ 33-item Feelings of Stigmatization Questionnaire } \\
\hline$r$ & 0.018 & 0.414 & - & - \\
\hline$p$-Value & 0.736 & $<0.001^{*}$ & - & - \\
\hline \multicolumn{5}{|l|}{ SWLS } \\
\hline$r$ & 0.032 & - & - & - \\
\hline$p$-Value & 0.544 & - & - & - \\
\hline
\end{tabular}

AIS Acceptance of Illness Scale, DLQI Dermatology Life Quality Index, $r$ Spearman's correlation coefficient, SWLS Satisfaction with Life Scale

* Statistically significant correlation

respondents aged 41-50 years, whereas Jankowiak et al. [23] found the lowest levels of satisfaction with life among 30-39-year-old patients.

In our present study, the mean score for the 33-item Feelings of Stigmatization Questionnaire was 90.06 pts, as compared with 84.3 pts in the study conducted by Hawro et al. [24]. Mean score of our respondents on the 6-item Stigmatization Scale was 8.73 pts, which is a substantially higher value than the mean stigmatization level reported for patients from the United Arab Emirates (5.6 pts) [25] and the

Fig. 4 Scatterplots illustrating correlations between standardized research instruments AIS Acceptance of Illness Scale, DLQI Dermatology Life Quality Index, SWLS Satisfaction with Life Scale 

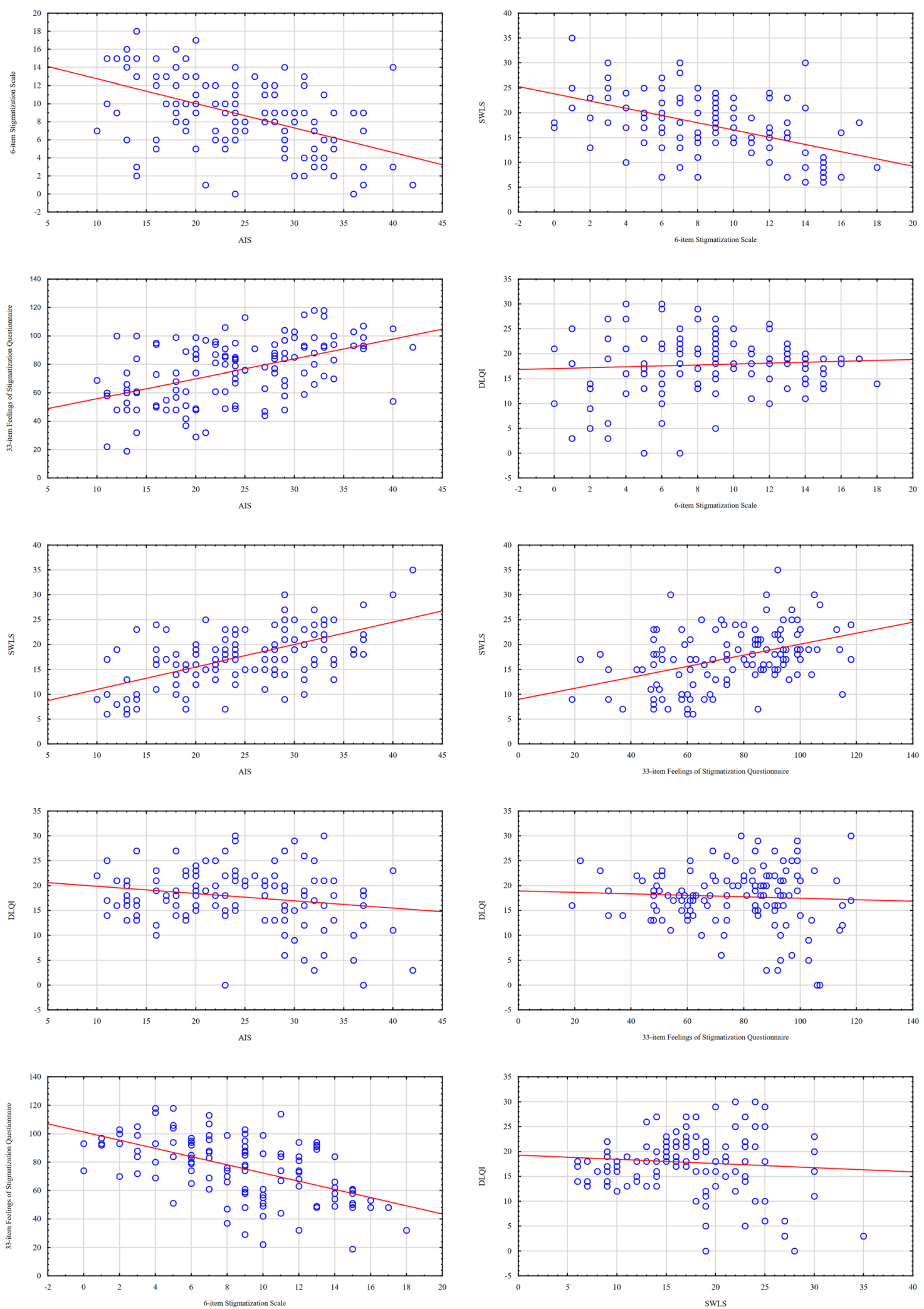
mean score documented in another study of Polish people with psoriasis (5.0 pts) [26].

We did not find statistically significant differences in the stigmatization scores of females and males with psoriasis. The results of previous studies analyzing the relationship between sex and stigmatization level in psoriasis are inconclusive. Some authors found higher stigmatization levels among women [27-29], whereas others did not observe significant sex-related differences in this parameter [16]. Similar to our study, Hrehorów et al. [30] did not find a statistically significant difference in the stigmatization levels of Polish females and males with psoriasis. The lack of significant sex-related differences in the stigmatization levels might be explained by the fact that a growing proportion of contemporary men pay attention to their image and want to be attractive [31, 32].

A growing body of evidence shows that psoriasis has a detrimental effect on quality of life [33, 34].

Mean quality of life score in the present study was slightly less than 18 pts. Mean DLQI score for 34 people with psoriasis participating in a study conducted by Martinez-Garcia et al. [35] was 12 pts. In a study carried out by Moradi et al. [36], among 62 Iranian patients with psoriasis, the mean DQLI score was only 8 pts. The mean DLQI in another study, conducted by Lin et al. [22], was 9.16 pts. In a study of Greek people with psoriasis, the mean DLQI score was 12.61 pts [37]. Kwan et al. [38] reported a mean DLQI score of 6 pts. In their study, the DLQI scores for $31 \%$ of respondents corresponded to a severe deterioration of quality of life. The mean DLQI score for patients surveyed by Jung et al. [39] was 12.4 pts, with $53.8 \%$ of participants showing severely deteriorated quality of life. The mean DLQI score for people with psoriasis who participated in a study conducted by An et al. [40] was 13.32 pts, with $39.4 \%$ of respondents scoring at least 11 pts. In our present study, the proportion of patients who scored 11 pts and above was $47 \%$.

We found a significant correlation between the level of illness acceptance and quality of life with psoriasis. Greater acceptance of the illness was associated with higher satisfaction with life, and a decrease in illness acceptance correlated with deterioration of quality of life. Published data on the relationship between illness acceptance and quality of life with psoriasis are inconclusive. While some authors did not find an association between these variables [41], others reported significant correlations between the two [42-44].

Age is an important determinant of quality of life with psoriasis. Previous studies demonstrated that psoriasis had a greater impact on physical health in older patients, whereas the effect of the disease in younger age groups was observed primarily with regards to psychical and social domains of health [45-47]. In our present study, younger age groups (especially patients aged 41-50 and 51-60 years) contained a smaller proportion of persons who assessed their quality of life as poor. These observations are consistent with the findings published by Lin et al. [22], as well as with the results of a study conducted by Zachariae et al. [46] among people with psoriasis from Scandinavian countries; in the latter study, older patients also showed better quality of life than respondents from younger age groups. However, some studies demonstrated an opposite relationship between age and quality of life; For example, a study conducted by Italian researchers showed that older people with psoriasis had worse quality of life than working-age patients [48].

We found that the proportion of women who assessed their quality of life as poor was larger than the respective proportion of male patients. Similar observations were also previously reported by Italian [48] and Turkish researchers [49]. Also, a study conducted in Japan showed that psoriasis had a more detrimental effect on quality of life in women than in men [50].

The present study has some potential limitations which need to be considered for the interpretation of its results. One of them is the cross-sectional design of the study and the fact that the data were collected solely with self-report questionnaires. Another potential limitation might be a relatively small sample size. Furthermore, the group of patients recruited at a single institution was not necessarily representative of the whole population of people with psoriasis, hence our results should not be 
generalized onto all patients. Finally, no comparison with a control group was included in the study protocol. Despite these potential limitations, the results of this study might constitute a starting point for further research on the influence of sociodemographic variables on illness acceptance, satisfaction with life, stigmatization, and quality of life with psoriasis. Optimally, these relationships should be the subject of a longitudinal study.

\section{CONCLUSIONS}

1. People with psoriasis participating in this study showed moderate levels of illness acceptance and stigmatization, low level of satisfaction with life, and moderately deteriorated quality of life.

2. Acceptance of illness was significantly modulated by patient sex. The level of illness acceptance among men was significantly higher than among women. Furthermore, illness acceptance exerted a significant effect on satisfaction with life with psoriasis.

3. Place of residence exerted significant effects on satisfaction with life (higher in countryside dwellers), sense of stigmatization (stronger in countryside dwellers), and quality of life (more severely deteriorated in city/town dwellers).

4. Duration of disease significantly affects the degree of life satisfaction, sense of stigmatization (measured using a 33-item Feelings of Stigmatization Questionnaire), and quality of life among patients with psoriasis.

\section{ACKNOWLEDGEMENTS}

The authors would like to express their gratitude to the cooperating institution and all participants.

Funding. The journal's Rapid Service Fee was funded by the Medical University of Białystok. The study was funded by the authors.
Authorship. All named authors meet the International Committee of Medical Journal Editors (ICMJE) criteria for authorship for this article, take responsibility for the integrity of the work as a whole, and have given their approval for this version to be published.

Disclosures. Beata Kowalewska, Mateusz Cybulski, Barbara Jankowiak, and Elżbieta Krajewska-Kułak have nothing to disclose.

Compliance with Ethics Guidelines. The study protocol was approved by the Bioethics Committee of the Medical University in Białystok, in line with the relevant Polish law (statute no. R-I-002/285/2018 of 28 July 2018). The study procedures were carried out in agreement with the Declaration of Helsinki, as revised in 2018, and good clinical practice guidelines. Written informed consent to participate was obtained from all patients before enrollment.

Data Availability. The datasets generated and analyzed in the current study are available from the corresponding author on reasonable request.

Open Access. This article is licensed under a Creative Commons Attribution-NonCommercial 4.0 International License, which permits any non-commercial use, sharing, adaptation, distribution and reproduction in any medium or format, as long as you give appropriate credit to the original author(s) and the source, provide a link to the Creative Commons licence, and indicate if changes were made. The images or other third party material in this article are included in the article's Creative Commons licence, unless indicated otherwise in a credit line to the material. If material is not included in the article's Creative Commons licence and your intended use is not permitted by statutory regulation or exceeds the permitted use, you will need to obtain permission directly from the copyright holder. To view a copy of this licence, visit http://creativecommons.org/licenses/bync/4.0/. 


\section{REFERENCES}

1. Inanir I, Aydemir O, Gündüz K, Danaci AE, Türel A. Developing a quality of life instrument in patients with psoriasis: the Psoriasis Quality of Life Questionnaire (PQLQ). Int J Dermatol. 2006;45:234-8.

2. Langley RG, Krueger GG, Griffiths CE. Psoriasis: epidemiology, clinical features, and quality of life. Ann Rheum Dis. 2005;64(Suppl 2):18-23.

3. Bhosle MJ, Kulkarni A, Feldman SR, Balkrishnan R. Quality of life in patients with psoriasis. Health Qual Life Outcomes. 2006;4:35.

4. Korman NJ, Zhao Y, Pike J, Roberts J. Relationship between psoriasis severity, clinical symptoms, quality of life and work productivity among patients in the USA. Clin Exp Dermatol. 2016;41: 514-21.

5. Augustin M, Krüger K, Radtke MA, Schwippl I, Reich $\mathrm{K}$. Disease severity, quality of life and health care in plaque-type psoriasis: a multicenter cross-sectional study in Germany. Dermatology. 2008;216:366-72.

6. Korman NJ, Zhao Y, Pike J, Roberts J, Sullivan E. Increased severity of itching, pain, and scaling in psoriasis patients is associated with increased disease severity, reduced quality of life, and reduced work productivity. Dermatol Online J. 2015;21: 13030/qt1x16v3dg.

7. Zill JM, Dirmaier J, Augustin M, Dwinger S, Christalle E, Härter $M$, et al. Psychosocial distress of patients with psoriasis: protocol for an assessment of care needs and the development of a supportive intervention. JMIR Res Protoc. 2018;7:e22.

8. Kostyła M, Tabała K, Kocur J. Illness acceptance degree versus intensity of psychopathological symptoms in patients with psoriasis. Postepy Dermatol Alergol. 2013;30:134-9.

9. Fortune DG, Richards HL, Main CJ, Griffiths CE. Patients' strategies for coping with psoriasis. Clin Exp Dermatol. 2002;27:177-84.

10. Randa H, Todberg T, Skov L, Larsen LS, Zachariae R. Health-related quality of life in children and adolescents with psoriasis: a systematic review and meta-analysis. Acta Derm Venereol. 2017;97: 555-63.

11. Kwan Z, Bong YB, Tan LL, Lim SX, Yong ASW, Ch'ng CC, et al. Determinants of quality of life and psychological status in adults with psoriasis. Arch Dermatol Res. 2018;310:443-51.

12. Randa H, Lomholt JJ, Skov L, Zachariae R. Healthrelated quality of life in adolescents with psoriasis: an interview-based study. Br J Dermatol. 2018;178: 1404-11.

13. Juczyński Z. Narzędzia pomiaru w promocji i psychologii zdrowia. Warsaw: Pracownia Testów Psychologicznych Polskiego Towarzystwa Psychologicznego; 2012.

14. Felton BJ, Revenson TA, Hinrichsen GA. Stress and coping in the explanation of psychological adjustment among chronically ill adults. Soc Sci Med. 1984;18:889-98.

15. Diener E, Emmons RA, Larsen RJ, Griffin S. The satisfaction with life scale. J Pers Assess. 1985;49: $71-5$.

16. Lu Y, Duller P, van der Valk PG, Evers AW. Helplessness as predictor of perceived stigmatization in patients with psoriasis and atopic dermatitis. Dermatol Psychosom. 2003;4:146-50.

17. Hrehorów E, Szepietowski J, Reich A, Evers AW, Ginsburg IH. Instruments for stigmatization evaluation in patients suffering from psoriasis: Polish language versions. Dermatol Klin. 2006;8:253-8.

18. Ginsburg IH, Link BG. Feelings of stigmatization in patients with psoriasis. J Am Acad Dermatol. 1989;20:53-63.

19. Finlay AY, Khan GK. Dermatology Life Quality Index (DLQI): a simple practical measure for routine clinical use. Clin Exp Dermatol. 1994;19: 210-6.

20. Szepietowski J, Salomon J, Finlay AY, Klepacki A, Chodynicka B, Marionneau N, et al. Dermatology Life Quality Index (DLQI): Polish version. Dermatol Klin. 2004;6:63-70.

21. Hongbo Y, Thomas CL, Harrison MA, Salek MS, Finlay AY. Translating the science of quality of life into practice: what do Dermatology Life Quality Index scores mean? J Investig Dermatol. 2005;25: 659-64.

22. Lin TY, See LC, Shen YM, Liang CY, Chang HN, Lin YK. Quality of life in patients with psoriasis in northern Taiwan. Chang Gung Med J. 2011;34: 186-96.

23. Jankowiak B, Sekmistrz S, Kowalewska B, Niczyporuk W, Krajewska-Kułak E. Satisfaction with life in a group of psoriasis patients. Postepy Dermatol Alergol. 2013;30:85-90.

24. Hawro M, Maurer M, Weller K, Maleszka R, Zalewska-Janowska A, Kaszuba A, et al. Lesions on the back of hands and female gender predispose to stigmatization in patients with psoriasis. J Am Acad Dermatol. 2017;76:648-54. 
25. Dimitrov D, Matusiak $Ł$, Szepietowski JC. Stigmatization in Arabic psoriatic patients in the United Arab Emirates-a cross sectional study. Postepy Dermatol Alergol. 2019;36:425-30.

26. Jankowiak B, Kowalewska B, Fiodaravich Khvorik D, Krajewska-Kułak E, Niczyporuk W. The level of stigmatization and depression of patients with psoriasis. Iran J Public Health. 2016;45:690-2.

27. Zięciak T, Rzepa T, Król J, Żaba R. Stigmatization feelings and depression symptoms in psoriasis patients. Psychiatr Pol. 2017;51:1153-63.

28. Schmid-Ott G, Schallmayer S, Calliess IT. Quality of life in patients with psoriasis and psoriasis arthritis with a special focus on stigmatization experience. Clin Dermatol. 2007;25:547-54.

29. Hawro T, Janusz I, Zalewska A, Miniszewska J. Jakość życia i stygmatyzacja a nasilenie zmian skórnych i świądu u osób chorych na łuszczycę. In: Rzepa T, Szepietowski J, Żaba R, editors. Psychologiczne i medyczne aspekty chorób skóry. Wrocław: Cornetis; 2011. p. 42-51.

30. Hrehorów E, Salomon J, Matusiak $Ł$, Reich A, Szepietowski JC. Patients with psoriasis feel stigmatized. Acta Derm Venereol. 2012;92:67-72.

31. Makowska I, Gmitrowicz A. Psychodermatologythe interface between dermatology, psychiatry and psychology. Psychiatr Psychol Klin. 2014;14:100-5.

32. Renzetti CM, Curran DJ. Kobiety, mężczyźni i społeczeństwo. Warsaw: Wydawnictwo Naukowe PWN; 2005.

33. Basra MK, Sue-Ho R, Finlay AY. The Family Dermatology Life Quality Index: measuring the secondary impact of skin diseases. Br J Dermatol. 2007;156:528-38.

34. Richards HL, Fortune DG, Griffiths CE. The greater patient concept. Br J Dermatol. 2008;158:176-8.

35. Martínez-García E, Arias-Santiago S, ValenzuelaSalas I, Garrido-Colmenero C, García-Mellado V, Buendía-Eisman A. Quality of life in persons living with psoriasis patients. J Am Acad Dermatol. 2014;71:302-7.

36. Moradi M, Rencz F, Brodszky V, Moradi A, Balogh $\mathrm{O}$, Gulácsi L. Health status and quality of life in patients with psoriasis: an Iranian cross-sectional survey. Arch Iran Med. 2015;18:153-9.

37. Kouris A, Christodoulou C, Stefanaki C, Livaditis M, Tsatovidou R, Kouskoukis C, et al. Quality of life and psychosocial aspects in Greek patients with psoriasis: a cross-sectional study. An Bras Dermatol. 2015;90:841-5.

38. Kwan Z, Bong YB, Tan LL, Lim SX, Yong AS, Ch'ng $\mathrm{CC}$, et al. Socioeconomic and sociocultural determinants of psychological distress and quality of life among patients with psoriasis in a selected multiethnic Malaysian population. Psychol Health Med. 2017;22:184-95.

39. Jung S, Lee SM, Suh D, Shin HT, Suh DC. The association of socioeconomic and clinical characteristics with health-related quality of life in patients with psoriasis: a cross-sectional study. Health Qual Life Outcomes. 2018;16:180.

40. An JG, Liu YT, Xiao SX, Wang JM, Geng SM, Dong YY. Quality of life of patients with neurodermatitis. Int J Med Sci. 2013;10:593-8.

41. Fortune DG, Main CJ, O'Sullivan TM, Griffiths CEM. Quality of life in patients with psoriasis: the contribution of clinical variables and psoriasisspecific stress. Br J Dermatol. 1997;137:755-60.

42. Rapp SR, Feldman SR, Excum ML, Fleischer AB, Reboussin DM. Psoriasis causes as much disability as other major medical diseases. J Am Acad Dermatol. 1999;41:401-6.

43. Rapp SR, Cottrell CA, Leary MR. Social coping strategies associated with quality of life decrements among psoriasis patients. Br J Dermatol. 2001;145: 610-6.

44. Uttjek M, Dufaker M, Nygren L, Stenberg B. Determinants of quality of life in a psoriasis population in northern Sweden. Acta Derm Venereol. 2004;84: 37-43.

45. Krueger G, Koo J, Lebwohl M, Menter A, Stern RS, Rolstad T. The impact of psoriasis on quality of life: results of a 1998 National Psoriasis Foundation patient membership survey. Arch Dermatol. 2001;137:280-4.

46. Zachariae R, Zachariae H, Blomqvist K, Davidsson S, Molin L, Mørk C, et al. Quality of life in 6497 Nordic patients with psoriasis. Br J Dermatol. 2002;146:1006-16.

47. Lundberg L, Johannesson M, Silverdahl M, Hermansson C, Lindberg M. Health-related quality of life in patients with psoriasis and atopic dermatitis measured with SF-36, DLQI and a subjective measure of disease activity. Acta Derm Venereol. 2000;80:430-4. 
48. Sampogna F, Chren MM, Melchi CF, Pasquini P, Tabolli S, Abeni D. Age, gender, quality of life and psychological distress in patients hospitalized with psoriasis. Br J Dermatol. 2006;154:325-31.

49. Çakmur H, Derviş E. The relationship between quality of life and the severity of psoriasis in Turkey. Eur J Dermatol. 2015;25:169-76.
50. Mabuchi T, Yamaoka H, Kojima T, Ikoma N, Akasaka E, et al. Psoriasis affects patient's quality of life more seriously in female than in male in Japan. Tokai J Exp Clin Med. 2012;37:84-8. 